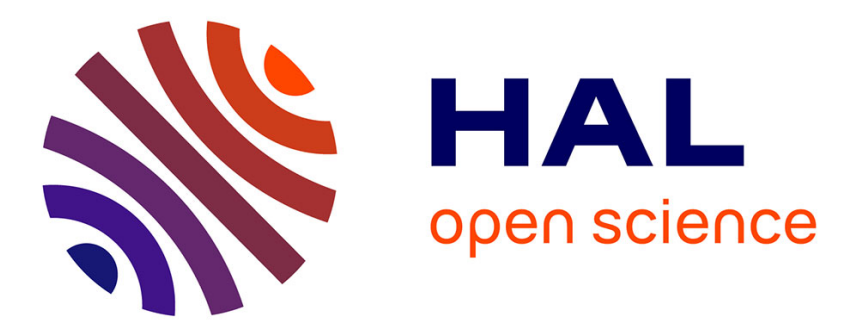

\title{
Structure cristallines de deux variétés allotropiques de manganite de baryum. Nouvelle structure ABO3
}

\author{
Antoine Hardy
}

\section{To cite this version:}

Antoine Hardy. Structure cristallines de deux variétés allotropiques de manganite de baryum. Nouvelle structure ABO3. Acta Crystallographica [1948-1967], 1962, 15, pp.179-181. 10.1107/S0365110X6200047X . hal-00124003

\section{HAL Id: hal-00124003 \\ https://hal.science/hal-00124003}

Submitted on 13 Jun 2013

HAL is a multi-disciplinary open access archive for the deposit and dissemination of scientific research documents, whether they are published or not. The documents may come from teaching and research institutions in France or abroad, or from public or private research centers.
L'archive ouverte pluridisciplinaire HAL, est destinée au dépôt et à la diffusion de documents scientifiques de niveau recherche, publiés ou non, émanant des établissements d'enseignement et de recherche français ou étrangers, des laboratoires publics ou privés. 


\title{
Structures Gristallines de Deux Variétés Allotropiques de Manganite de Baryum. Nouvelle Structure $\mathrm{ABO}_{3}$
}

\author{
Par Antoine Hardy \\ Service de Chimie Minérale. Faculté des Sciences de Bordeaux; Commissariat á l'Energie Atomique, \\ Service de Physique des Solides, France
}

(Reçu le 2 juin 1961 et révisé le 27 juin 1961)

\begin{abstract}
$\mathrm{BaMnO}_{3}$ at low temperature is hexagonal from the type $\mathrm{BaNiO}_{3}$. The space group is $C_{6 v}^{4}$, the parameters are $a=5 \cdot 672$ and $c=4 \cdot 71 \AA$. There are two $A B \mathrm{O}_{3}$ units in a unit cell. $\mathrm{BaMnO}_{3}$ at high temperature belongs to the space group $D_{6 h}^{4}$ with $a=5 \cdot 669$ and $c=9 \cdot 38 \AA$. The hexagonal unit cell has four sample $\mathrm{ABO}_{3}$ units. The lattice is constituted by layers of composition $\mathrm{BaO}_{3}$ in a close-packed arrangement of atoms, like the one of the perowskite, but with a sequence $A B A C$.
\end{abstract}

Les composés, de formule générale $A B \mathrm{O}_{3}$, dérivés du type perowskite ont fait l'objet de nombreux travaux. Les différentes structures mentionnées correspondent à $1,2,6,8$ ou même davantage de motifs $A B \mathrm{O}_{3}$ par maille.

Burbank (1948) envisage toutefois la possibilité d'une structure à 4 motifs.

\section{$\mathrm{BaMnO}_{3}$ de basse température}

Le manganite $\mathrm{BaMnO}_{3}$ de basse température a été préparé suivant une méthode analogue à celle de Scholder (1952), par thermolyse du manganate $\mathrm{BaMnO}_{4}$. La réaction est complète dès $400{ }^{\circ} \mathrm{C}$. (Hardy, 1961). Nous avons vérifié oxydimétriquement que le manganèse répond à la valence 4 dans le produit formé.
L'étude radiocristallographique a été faite avec le rayonnement $\alpha$ du fer sur le produit pulvérulent. La similitude des diagrammes $X$ montre que $\mathrm{BaMnO}_{3}$ de basse température est un isotype de $\mathrm{BaNiO}_{3}$ dont la structure a été établie par Lander (1951). Le groupe d'espace est $P 6_{3} m c, C_{6 v}^{4}$; les paramètres de la maille hexagonale ont pour valeurs:

d'où :

$$
a=5,672 \pm 0,004, c=4,71 \pm 0,01 \AA
$$

$$
c / a=0,830 \text {. }
$$

La présence de 2 motifs $\mathrm{BaMnO}_{3}$ dans la maille donne une densité $X\left(d_{x}=6,01 \mathrm{~g} . / \mathrm{ml}\right.$.) très voisine de la densité observée au picnomètre $(d=6,06 \pm 0,10$ g./ml.).

L'atome de manganèse se trouve en position $(a)$ 
avec $z=0$, le baryum en position (b) avec $z=\frac{1}{4}$ et l'oxygène en position $(c)$ avec $x=\frac{1}{6}$ et $z=\frac{3}{4}$, ce qui donne la répartition:

$\begin{array}{lll}\text { Mn } & 2 & 0,0,0 \\ \mathrm{Ba} & 2 & \frac{1}{3}, \frac{2}{3}, \frac{1}{4} \\ \mathrm{O} & 6 & \frac{1}{6}, \frac{1}{6}, \frac{3}{4}\end{array}$

Pour le calcul des intensités de diffraction $X$ nous avons utilisé les facteurs de structure des Tables Internationales. Le Tableau 1 rassemble les indices de Miller possibles $(h k \cdot l)$, les distances observées $\left(d_{o}\right)$, les intensités calculées $\left(I_{c}\right)$ et celles observées $\left(I_{o}\right)$ déterminées au microdensitomètre Vassy. La concordance des intensités justifie les positions des atomes.

Tableau 1. Données pour $\mathrm{BaMnO}_{3}$ de basse température

\begin{tabular}{|c|c|c|c|c|c|c|}
\hline$d_{o}(\AA)$ & $I_{c}$ & $I_{o}$ & $h k \cdot l$ & $d_{o}(\AA)$ & $I_{c}$ & $I_{o}$ \\
\hline $10 \cdot 0$ & $<1$ & & $30 \cdot 2$ & 1,325 & 25 & 20 \\
\hline 3,40 & 88 & 80 & $20 \cdot 3\}$ & & 13) & \\
\hline 2,83 & 100 & 100 & $31 \cdot 1\}$ & 1,312 & $16\}$ & 20 \\
\hline $20 \cdot 0$ & 3 & & $40 \cdot 0$ & & $<1$ & \\
\hline 2,36 & 9 & 10 & $22 \cdot 2$ & 1,212 & 9 & 10 \\
\hline 218 & 69) & 90 & $21 \cdot 3)$ & & 14 ) & \\
\hline 2,18 & $28\}$ & & $40 \cdot 1$ & & 14 & \\
\hline ter & $<1$ & & $31 \cdot 2\}$ & 1,100 & 15 & $3 x$ \\
\hline 1,808 & 16 & 20 & $00 \cdot 4)$ & & 5) & \\
\hline & $30)$ & 40 & $32 \cdot 0$ & & $<1$ & \\
\hline 1,731 & $24\}$ & 40 & $10 \cdot 4$ & & $<1$ & \\
\hline 1,639 & 18 & 20 & $32 \cdot 1$ & & 16 & \\
\hline & 2 & & $40 \cdot 2\}$ & 1,094 & 12 & 4 \\
\hline & 3 & & 11.4 & & 18) & \\
\hline 1,418 & 20 & 20 & $41 \cdot 0$ & 1,072 & 19 & 16 \\
\hline
\end{tabular}

Le baryum, qui a douze voisins oxygène, constitue avec ceux-ci un empilement compact d'atomes. Le manganèse est situé au centre d'octaèdres réguliers formés d'oxygènes et groupés par files avec faces communes. Les distances inter-atomiques sont:

$$
\begin{array}{ll}
\mathrm{Ba}-\mathrm{O}_{1}=2,84 \AA & \mathrm{Ba}-\mathrm{O}_{2}=2,86 \AA \\
\mathrm{Mn}-\mathrm{O}=2,02 \AA & \mathrm{Mn}-\mathrm{Mn}=2,35 \AA
\end{array}
$$

\section{$\mathrm{BaMnO}_{3}$ de haute température}

$\mathrm{La}$ thermolyse du manganite de baryum $\mathrm{BaMnO}_{3}$ au-dessus de $1150{ }^{\circ} \mathrm{C}$. conduit à une nouvelle variété allotropique. On observe sur le spectre de poudre des raies aux mêmes espacements que sur le spectre de la forme de basse température et, de plus, de nouvelles raies d'intensités moyennes et faibles. L'analyse oxydimétrique confirme la valence $4 \mathrm{du}$ manganèse.

Pour obtenir des monocristaux de $\mathrm{BaMnO}_{3}$ de haute température, nous avons chauffé un mélange de carbonates de baryum et de manganèse dans un rapport moléculaire de 4 à 1 , sous argon à $1300{ }^{\circ} \mathrm{C}$. pendant 10 heures; le refroidissement est lent. La variété obtenue se présente sous forme de petites paillettes noires, hexagonales et prismatiques.

Les diagrammes de Laue indiquent la présence d'un axe sénaire orthogonal à deux séries d'axes binaires. La méthode du cristal tournant donne les paramètres.
La masse spécifique, mesurée picnométriquement $(\varrho=6,07 \pm 0,10$ g. $/ \mathrm{ml}$.), et le volume de la maille imposent à celle-ci 4 motifs $\mathrm{BaMnO}_{3}$.

Le groupe d'espace est déterminé sans ambiguité par les extinctions systématiques relevées sur les diagrammes de Weissenberg obtenus avec le rayonnement $\alpha$ du fer pour les différentes zones accessibles selon les deux axes binaires différenciés: $P 6_{3} / m m c$, $D_{6 h}^{4}$.

Le spectre de poudre Debye-Scherrer permet de préciser les paramètres:

$$
a=5,669 \pm 0,003, c=9,375 \pm 0,015 \AA
$$

d'où le rapport d'axes:

$$
c / a=1,637 .
$$

Cette valeur est très voisine de la valeur théorique pour un empilement compact d'atomes.

D'autre part, les taches $h k \cdot l$ n'existent, pour $h-k=$ $3 n$, que si $l=2 n$. Cette règle d'extinction spéciale, ainsi que la composition $\mathrm{BaO}_{3}$ des couches impliquent que:

(1) Le baryum occupe en $(a)$ et en (c) deux positions sans paramètre.

(2) Les oxygènes se situent en deux positions: l'une en $(g)$ sans paramètre, l'autre en $(h)$ avec $x=\frac{\overline{1}}{6}$.

(3) Le manganèse occupe en $(f)$ une position à un paramètre.

La place du manganèse a été précisée par projection axiale de la densité électronique selon les plans $(00 \cdot l)$. Le facteur de structure a été calculé au moyen de l'électro-sommateur de Fourier (Tournarie, à publier). L'intensité de la tache sensible $00 \cdot 6$, comparée à celles pratiquement constantes des taches $00 \cdot 4$ et $00 \cdot 8$, donne pour $z$ la valeur 0,61 (Fig. 1). La répartition dans la maille est:

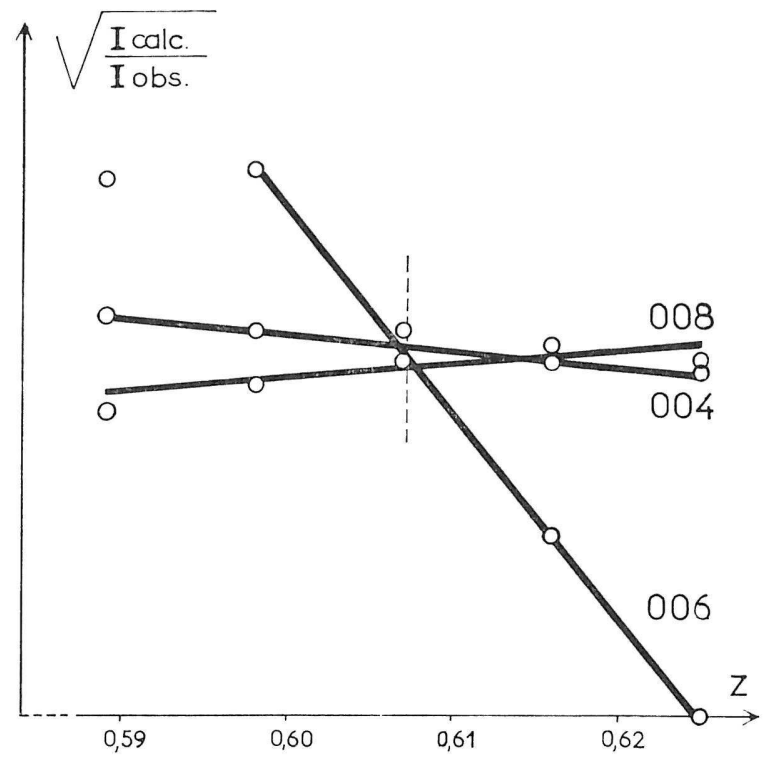

Fig. 1. Recherche de la position du manganèse par comparaison des intensités des taches $00 \cdot l$. 


$\begin{array}{lllll}\mathrm{Ba} & 2 & 0,0,0 & 2 & \frac{1}{3}, \frac{2}{3}, \frac{1}{4} \\ \mathrm{Mn} & 4 & \frac{1}{3}, \frac{2}{3}, 0,61 & & \\ \mathrm{O} & 6 & \frac{1}{2}, 0,0 & 6 & \frac{\overline{1}}{6}, \frac{\overline{2}}{6}, \frac{1}{4}\end{array}$

Pour le calcul des intensités de diffraction $X$, nous avons utilisé les facteurs de structure des Tables Internationales. Le facteur de diffusion de $\mathrm{Mn}^{4+}$ a été calculé par interpolation de ceux de $\mathrm{Mn}^{0}$ et de $\mathrm{Mn}^{7+}$. Ce dernier a été extrapolé à partir des facteurs de diffusion des ions possédant le même nombre d'électrons. Le Tableau 2 groupe les indices de Miller, les distances réticulaires observées, les intensités calculées et observées.
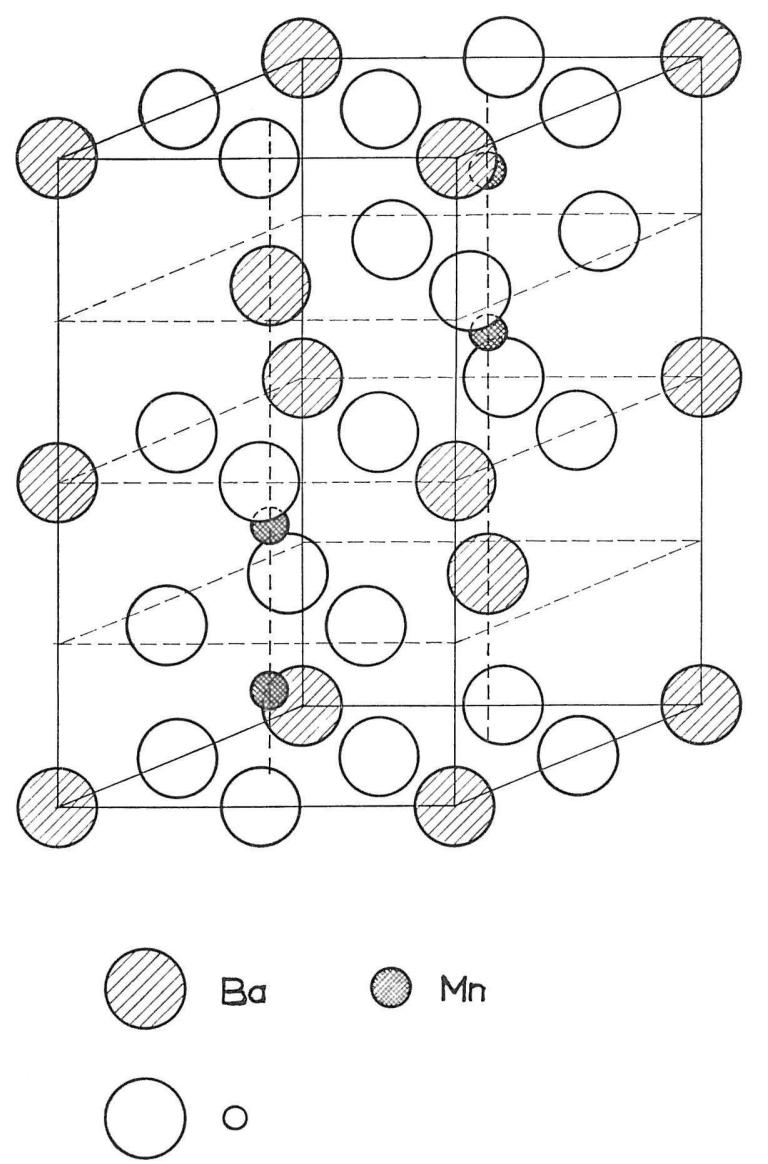

Fig. 2. Structure de $\mathrm{BaMnO}_{3}$ de haute température. La maille de $\mathrm{BaMnO}_{3}$ de basse température peut-être représentée par la moitié inférieure ou supérieure de cette figure.

Dans l'empilement compact de composition $\mathrm{BaO}_{3}$ les couches se succèdent selon la suite $A B A C$. Celle-ci est intermédiaire entre la succession $A B$, qui existe dans $\mathrm{BaMnO}_{3}$ de basse température, et la succession $A B C$ de l'empilement cubique.

Chaque manganèse est presqu'au centre d'un octaèdre d'oxygènes. Ces octaèdres sont groupés par deux, avec une face commune, au lieu d'être en files, comme pour la variété précédente, ou isolés, comme
Tableau 2. Données pour $\mathrm{BaMnO}_{3}$ de haute température

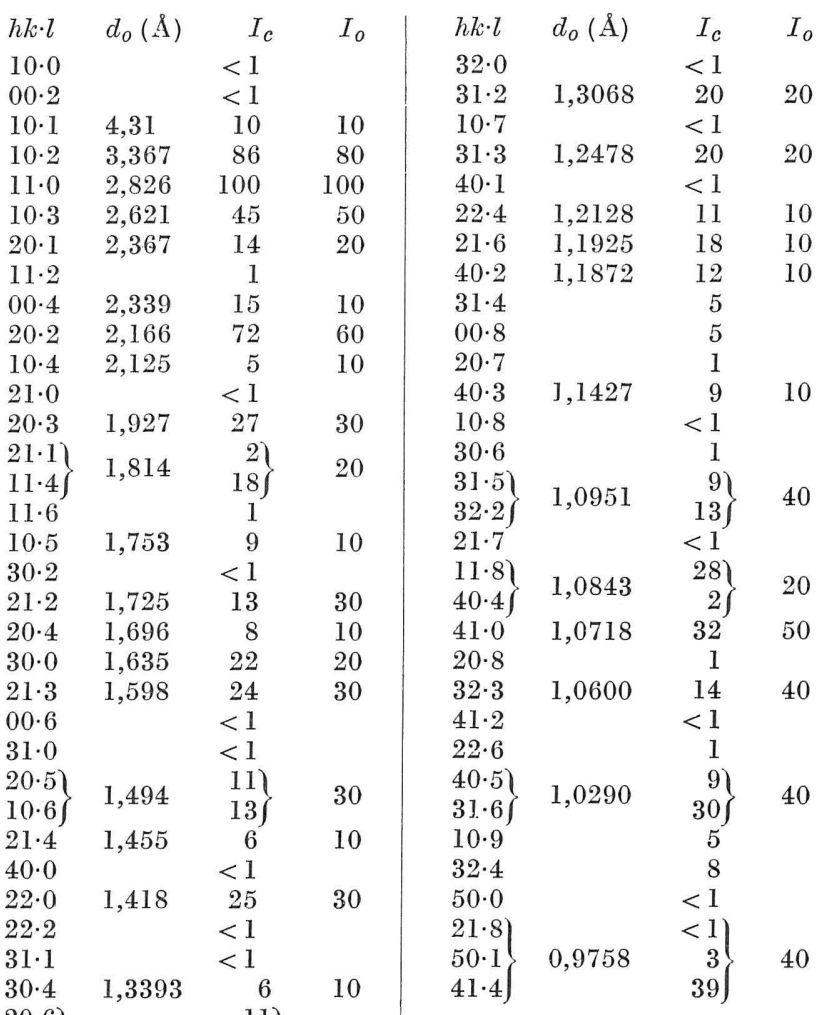

.

dans un édifice de type perowskite cubique (Fig. 2).

Le titanate de baryum $\mathrm{BaTiO}_{3}$ de haute température (Burbank \& Evans, 1948) possède également une structure intermédiaire; mais celle-ci est plus voisine du type cubique, l'empilement compact des couches constituant une suite $A B C A C B$ et deux octaèdres seulement sur trois étant jumelés.

Les distances inter-atomiques relatives à $\mathrm{BaMnO}_{3}$ de haute température sont très voisines de celles de la première variété:

$$
\begin{aligned}
& \mathrm{Ba}-\mathrm{O}_{1}=2,83 \AA \quad \mathrm{Ba}-\mathrm{O}_{2}=2,86 \AA \\
& \mathrm{Mn}-\mathrm{O}_{1}=1,94 \AA \quad \mathrm{Mn}-\mathrm{O}_{2}=2,10 \AA \\
& \mathrm{Mn}_{1}-\mathrm{Mn}_{2}=2,62 \AA \quad \mathrm{Mn}_{1}-\mathrm{Mn}_{2}{ }^{\prime}=3,88 \AA
\end{aligned}
$$

En résumé, la structure de $\mathrm{BaMnO}_{3}$ de basse température répond au groupe d'espace $C_{6 v}^{4}$; il est isotype de $\mathrm{BaNiO}_{3}$. La variété de haute température appartient au groupe $D_{6 h}^{4}$. Elle est le premier exemple, à notre connaissance, d'une structure $\mathrm{ABO}_{3}$ à 4 motifs élémentaires.

\section{Références}

Burbank, R. D. \& Evans, H. T., Jr. (1948). Acta Cryst. 1,330 .

Hardy, A. (1961). Bull. Soc. Chim. Franç. 7, 1329.

LANDER, J. J. (1951). Acta Cryst. 4, 152.

SCHOLdER, R. (1952). Z. für Elektrochemie, 56, 880.

TournarIE, M. (À publier.) 
Printed in Denmark at Fr. Bagges kgl. Hofbogtrykkeri, Copenhagen 